\title{
Stage-Specific Effects of Fibroblast \\ Growth Factor 2 on the Differentiation of Dental Pulp Cells
}

\author{
Karen Sagomonyants Mina Mina \\ Department of Craniofacial Sciences, School of Dental Medicine, University of Connecticut Health Center, \\ Farmington, Conn., USA
}

\section{Key Words}

Odontoblast differentiation - Dental pulp · Progenitors ·

Fibroblast growth factor 2 - Green fluorescent protein

\begin{abstract}
Dentinogenesis is a complex and multistep process, which is regulated by various growth factors, including members of the fibroblast growth factor (FGF) family. Both positive and negative effects of FGFs on dentinogenesis have been reported, but the underlying mechanisms of these conflicting results are still unclear. To gain a better insight into the role of FGF2 in dentinogenesis, we used dental pulp cells from various transgenic mice, in which fluorescent protein expression identifies cells at different stages of odontoblast differentiation. Our results showed that the continuous exposure of pulp cells to FGF2 inhibited mineralization and revealed both the stimulatory and inhibitory effects of FGF2 on the expression of markers of dentinogenesis and various transgenes. During the proliferation phase of in vitro growth, FGF2 increased the expression of markers of dentinogenesis and the percentages of dentin matrix protein 1/green fluorescent protein (DMP1-GFP)-positive functional odontoblasts and dentin sialophosphoprotein (DSPP)-Ceruleanpositive odontoblasts. Additional exposure to FGF2 during the differentiation/mineralization phase of in vitro growth decreased the extent of mineralization and the expression of
\end{abstract}

markers of dentinogenesis and of the DMP1-GFP and DSPPCerulean transgenes. Recovery experiments showed that the inhibitory effects of FGF2 on dentinogenesis were related to the blocking of the differentiation of cells into mature odontoblasts. These observations together showed the stage-specific effects of FGF2 on dentinogenesis by dental pulp cells, and they provide critical information for the development of improved treatments for vital pulp therapy and dentin regeneration.

(c) 2015 S. Karger AG, Basel

\section{Abbreviations used in this paper}

BMSC bone marrow stromal cell

DMP1 dentin matrix protein 1

DSPP dentin sialophosphoprotein

FACS flow-cytometric sorting

FBS fetal bovine serum

FGF fibroblast growth factor

FGFR fibroblast growth factor receptor

GFP green fluorescent protein

aMEM Minimum Essential Medium alpha

qPCR quantitative PCR

RT room temperature

$\mathrm{VH} \quad$ vehicle

$\mathrm{XO} \quad$ Xylenol Orange

\section{KARGER}

E-Mail karger@karger.com

www.karger.com/cto
(C) 2015 S. Karger AG, Basel

$1422-6405 / 15 / 1996-0311 \$ 39.50 / 0$
Mina Mina

Division of Pediatric Dentistry, Department of Craniofacial Sciences School of Dental Medicine, University of Connecticut Health Center Farmington, CT 06030 (USA)

E-Mail Mina@uchc.edu 


\section{Introduction}

Fibroblast growth factors (FGFs) are a family of signaling molecules shown to play essential roles in the development, repair and regeneration of damaged skeletal tissues [Hatch, 2010; Miraoui and Marie, 2010; Marie, 2012; Marie et al., 2012; Kim et al., 2014]. Currently, the FGF family contains 22 members, which elicit their effects through interaction with four highly conserved transmembrane tyrosine kinase receptors, i.e. FGF receptor (FGFR)1-4, in concert with heparin or heparan sulfate proteoglycans [Hatch, 2010; Miraoui and Marie, 2010]. Three major downstream signaling pathways which mediate the effects of FGF/FGFR signaling on cellular processes include MAPK, PI3K/Akt and PLC $\gamma$ [Miraoui and Marie, 2010; Marie, 2012; Marie et al., 2012].

FGF signaling plays an essential role in osteogenesis and dentinogenesis, and FGF2, in particular, is widely expressed in the cells of odontoblast and osteoblast lineages and has been identified as a potent regulator of mineralization in vivo and in vitro [Roberts-Clark and Smith, 2000; Madan and Kramer, 2005; Cooper et al., 2010; Miraoui and Marie, 2010; Marie, 2012; Marie et al., 2012; Smith et al., 2012].

Earlier studies on osteogenic cells reported conflicting effects of FGF signaling on osteoblast differentiation and the production of mineralized matrix [Miraoui and Marie, 2010; Marie, 2012; Marie et al., 2012]. Later studies showed that the effects of FGF signaling depend on the stage of osteoblast maturation. In immature osteoblasts, FGF signaling induces proliferation, leading to increased osteogenesis in the long term, whereas in mature osteoblasts, FGF signaling inhibits differentiation and mineralization [Miraoui and Marie, 2010; Marie, 2012; Marie et al., 2012].

FGF signaling has been shown to play an important role in primary and reparative dentinogenesis [Smith et al., 2012; Li et al., 2014]. FGF2 affects the proliferation, homing and migration of healthy and inflamed dental pulp cells [Nakao et al., 2004; Morito et al., 2009; Shimabukuro et al., 2009; Xiao et al., 2009; Osathanon et al., 2011; Suzuki et al., 2011; Kim et al., 2014]. FGF2 also increases the expression of markers of mesenchymal stem cells Oct4, Nanog and Rex1 and the percentage of STRO1positive cells in the dental pulp [Morito et al., 2009; Osathanon et al., 2011; Wu et al., 2012].

Although there appears to be general agreement as to the effects of FGF signaling on cell proliferation, there are conflicting results about its effects on dentinogenesis and mineralization. Several studies showed that FGF2 inhib- ited dentinogenesis and the expression of dentin sialophosphoprotein (Dspp) [Tsuboi et al., 2003; Shimabukuro et al., 2009; Xiao et al., 2009; Kim et al., 2010, 2014]. On the other hand, other studies showed that FGF2 stimulated the expression of $D s p p$ in vitro and the formation of osteodentin in vivo [Kikuchi et al., 2007; Ishimatsu et al., 2009; Kim et al., 2010, 2014].

Thus, the precise effects of FGF signaling on the differentiation of cells in the odontoblast lineage are still not well understood, and most likely involve multiple intraand extracellular mediators and differential responses of various cell populations [Dailey et al., 2005]. This is partially due to the lack of availability of stage-specific markers for studying progression of cells in the odontoblast lineage.

To gain a better understanding of the progression of progenitor cells in the odontoblast lineage, we used a series of green fluorescent protein (GFP) reporter transgenic mice that display stage-specific activation of transgenes during odontoblast differentiation in vivo and in vitro [Braut et al., 2003; Balic et al., 2010b; Balic and Mina, 2011]. These studies showed that 2.3-GFP and 3.6-GFP transgenes were activated at early stages of odontoblast differentiation (i.e. polarizing odontoblasts and prior to the expression of $D m p 1$ and $D s p p$ ) whereas dentin matrix protein 1/green fluorescent protein (DMP1-GFP) was first activated in functional/secretory odontoblasts (cells expressing Dmp1 and low levels of Dspp) [Balic et al., 2010b; Balic and Mina, 2011]. All three transgenes (2.3GFP, 3.6-GFP and DMP1-GFP) were also expressed at high levels in fully differentiated/mature odontoblasts, and their temporal and spatial patterns of expression mimicked those of endogenous transcripts and proteins [Balic et al., 2010b; Balic and Mina, 2011].

In addition, we have recently generated new transgenic mice using the bacterial artificial chromosome, which directs the expression of the DSPP-Cerulean transgene to functional and fully differentiated odontoblasts [unpubl. data]. Our in vivo and in vitro studies showed that the expression of the DSPP-Cerulean transgene was limited to odontoblasts and correlated closely with the expression of endogenous Dspp, and it can be used to identify fully differentiated odontoblasts in the heterogeneous pulp cultures [unpubl. data].

Here, we have used dental pulp cells from various transgenic mice which display the stage-specific activation of transgenes during odontoblast differentiation, in order to gain further insight into the effects of FGF2 on the mineralization and dentinogenesis of dental pulp cells. 


\section{Materials and Methods}

\section{Primary Dental Pulp Cultures}

All experimental protocols involving animal tissues were approved by the Institutional Animal Care and Use Committee, University of Connecticut Health Center. The coronal portions of the pulps from the first and second molars were isolated from 5- to 7-day-old hemizygous pOBCol3.6GFP (referred to as 3.6-GFP), pOBCol2.3GFP (referred to as 2.3-GFP), DMP1-GFP, DSPP-Cerulean and nontransgenic pulps as described previously [Balic et al., 2010a]. All mice were maintained in the CD1 background. After isolation, $8.75 \times 10^{4}$ cells $/ \mathrm{cm}^{2}$ were grown first in Dulbecco's modified Eagle's medium, 20\% fetal bovine serum (FBS), $2 \mathrm{mM}$ L-glutamine, $40 \mathrm{U} / \mathrm{ml}$ penicillin, $40 \mu \mathrm{g} / \mathrm{ml}$ streptomycin and 0.1 $\mu \mathrm{g} / \mathrm{ml}$ Fungizone (Invitrogen). Three days later, the medium was changed to Dulbecco's modified Eagle's medium containing 5\% FBS. At day 7, mineralization was induced by addition of Minimum Essential Medium alpha ( $\alpha \mathrm{MEM}$ ) medium, 5\% FBS, with 50 $\mu \mathrm{g} / \mathrm{ml}$ fresh ascorbic acid and $4 \mathrm{mM} \beta$-glycerophosphate. Medium was changed every other day.

\section{Primary Bone Marrow Stromal Cell Cultures}

Bone marrow stromal cells (BMSCs) were prepared from the femurs and tibiae of 5- to 7-day-old pulps as described before [Balic et al., 2010a]. Briefly, single-cell suspension was prepared from flushed marrows, plated at a density of $6.5 \times 10^{5} \mathrm{cells} / \mathrm{cm}^{2}$ and grown in aMEM containing $10 \% \mathrm{FBS}, 40 \mathrm{U} / \mathrm{ml}$ penicillin and 40 $\mu \mathrm{g} / \mathrm{ml}$ streptomycin. Three days later, the medium was changed to aMEM and 5\% FBS. At day 7, when the cells became confluent, the medium was switched to the mineralization-inducing medium containing aMEM, 5\% FBS, with $50 \mu \mathrm{g} / \mathrm{ml}$ fresh ascorbic acid and $4 \mathrm{~mm} \beta$-glycerophosphate. Medium was changed every other day.

\section{FGF2 Treatment of Primary Cultures}

Cultures were exposed to low molecular weight $(18 \mathrm{kDa})$ bovine FGF2 (R\&D Systems, Inc., Minneapolis, Minn., USA) or vehicle (VH, $0.1 \%$ BSA fraction V in PBS) every other day during the proliferation and differentiation/mineralization phases of in vitro growth (between days 3 and 21).

\section{Detection and Quantification of Mineralization in Cultures}

Mineralization in live cultures was examined by Xylenol Orange (XO) staining as described previously [Balic et al., 2010b]. The mean fluorescence intensity of XO staining was measured using a multidetection monochromator microplate reader (Safire ${ }^{2}$, Tecan, Research Triangle Park, N.C., USA) as described previously [Kuhn et al., 2010] with minor modifications. Fluorometric measurements were performed at $570 / 610 \mathrm{~nm}$ wavelength (excitation/emission) and a gain of 80 . The entire area of each well was read at a scan density of $6 \times 6$ regions (high-sensitivity flash mode). Background fluorescence for XO was measured using cultures from the preodontoblastic Q705 cell line [Priam et al., 2005] that lacks a mineralization potential. The background fluorescence values were subtracted from respective XO measurements.

Mineralization in fixed cultures was examined using a modified von Kossa silver nitrate staining protocol as described previously [Balic et al., 2010a]. After staining, cultures were rinsed and images were acquired using a scanner. The area of mineralization (black precipitate) in each well was quantified using NIH ImageJ software and is represented as the percentage of total area analyzed as described before [Balic et al., 2010a].

\section{Immunocytochemistry}

Pulp cells derived from the DSPP-Cerulean transgenic mice were treated with $\mathrm{VH}$ or FGF2 and processed for immunocytochemistry as described previously [Mulrooney et al., 2001] with some modifications. Cells were fixed with $3.7 \%$ formaldehyde in PBS for $4 \mathrm{~min}$ at room temperature (RT), incubated with $0.5 \%$ Triton $\mathrm{X}$ in PBS for 10 min at RT, blocked with $3 \%$ milk for $1 \mathrm{~h}$ at RT and then incubated with 1:1,000 dilution of anti-GFP Alexa Fluor 488-conjugated antibody (Molecular Probes, Invitrogen) in $0.3 \%$ Triton $\mathrm{X}$ in PBS overnight at $4^{\circ} \mathrm{C}$. In these cultures, the anti-GFP antibody binds specifically to the Cerulean fluorescent protein to enhance its visualization. The nuclei were stained with $1.0 \mu \mathrm{g} / \mathrm{ml}$ Hoechst 33342 dye (Invitrogen) for $15 \mathrm{~min}$ at RT. After staining, coverslips were mounted using Dako fluorescent mounting medium (Dako North America, Inc., Carpinteria, Calif., USA) and cultures were visualized under the microscope using filters for DAPI and GFPtpz for the detection of Hoechst 33342 and GFP, respectively.

The percentage of DSPP-Cerulean-positive odontoblasts in the cultures was calculated as the ratio of cells stained with anti-GFP antibody (DSPP-Cerulean-positive cells) to the total number of Hoechst-positive cells. In each experiment, approximately 20,00030,000 Hoechst-positive cells were counted from 20-40 different areas of VH- and FGF2-treated cultures. Negative controls included primary BMSC cultures derived from the DSPP-Cerulean littermates stained with anti-GFP antibody and primary dental pulp cultures derived from the DSPP-Cerulean littermates without anti-GFP antibody.

Digital Imaging and Epifluorescence Analysis of Cell Cultures

GFP expression in the cell cultures at various time points was examined using a Zeiss AxioObserver Z.1 microscope equipped with an AxioCam MRc digital camera and appropriate filters. Exposure times were adjusted for optimum imaging and kept consistent for each time point of the culture. Panoramic images of larger areas of the cultures were obtained using a computer-controlled motorized imaging workstation and a Zeiss AxioObserver Z.1 microscope.

\section{Fluorescence Intensity of GFP}

The mean fluorescence intensity of GFP transgenes in each well was measured as described for XO staining. Fluorometric measurement was performed at 483/525 $\mathrm{nm}$ wavelength (excitation/ emission) for the 2.3-GFP transgene and at 500/540 nm wavelength for the 3.6-GFP and DMP1-GFP transgenes (a gain of 80 for all three transgenes). Background fluorescence for GFP was measured using dental pulp cultures from nontransgenic littermates, and these values were subtracted from the respective GFP measurements. Fluorometric measurements were also obtained in DSPP-Cerulean cultures stained with anti-GFP antibody (500/540 nm wavelength and a gain of 80$)$ and Hoechst 33342 dye (343/483 $\mathrm{nm}$ wavelength and a gain of 70). Background fluorescence for GFP was measured using BMSC cultures from the DSPP-Cerulean littermates stained with anti-GFP antibody, and these values were subtracted from the respective GFP measurements. 
RNA Extraction and Quantitative PCR Analysis

Total RNA was isolated using TRIzol reagent (Invitrogen) followed by cDNA synthesis and TaqMan quantitative (q)PCR analysis. TaqMan primers for Bsp, Dmp1, Dspp, Gapdh, osteocalcin and type I collagen were purchased from Applied Biosystems (online suppl. table 1; see www.karger.com/doi/10.1159/000371343 for all online suppl. material). All qPCR reactions were run using $7900 \mathrm{HT}$ fast real-time PCR system (Applied Biosystems) under the following conditions: $50^{\circ} \mathrm{C}$ for $2 \mathrm{~min}, 95^{\circ} \mathrm{C}$ for $10 \mathrm{~min}, 40$ cycles with denaturation at $95^{\circ} \mathrm{C}$ for $15 \mathrm{~s}$ and extension at $60^{\circ} \mathrm{C}$ for $1 \mathrm{~min}$. Amplification efficiency was determined using internal standard curves derived from a purified amplicon diluted 2-fold (0.14-9.0 $\mathrm{ng}$ ), and was close to $100 \%$ for all qPCR reactions. We defined the acceptable range of CT values representing gene expression to be between 10 and 35 cycles, according to the manufacturer's recommendations (Applied Biosystems).

\section{WST-1 Cell Proliferation Assay}

Cell proliferation was examined by WST-1 rapid cell proliferation assay according to the manufacturer's instructions (EMD Millipore Corporation, Billerica, Mass., USA). The assay is based on the cleavage of the tetrazolium salt WST-1 to formazan by cellular mitochondrial dehydrogenases. An increase in the number of viable cells results in an increase in the amount of formazan dye formed. Pulp cells were cultured in a 96-well microtiter plate (17 $\times 10^{3}$ cells $\left./ \mathrm{cm}^{2}\right)$, treated with VH or FGF2 $(20 \mathrm{ng} / \mathrm{ml})$ starting at day $3(0 \mathrm{~h})$ and processed for WST-1 assay at 24, 48, 72 and $96 \mathrm{~h}$ after treatment. Cells were incubated with WST-1 reagent for $2 \mathrm{~h}$ at $37^{\circ} \mathrm{C}$ and the amount of the formazan dye produced was quantified by the optical density at $450 \mathrm{~nm}$, using a Synergy ${ }^{\mathrm{TM}} \mathrm{HT}$ multidetection microplate reader, and analyzed using Gen5 ${ }^{\mathrm{TM}} 1.09$ data analysis software (BioTek Instruments, Winooski, Vt., USA). Background absorbance levels were measured using wells without cells (culture medium only), and these values were subtracted from the respective $\mathrm{VH}$ and FGF2 values.

\section{Flow-Cytometric Analysis and Sorting}

Cells from 3.6-GFP, 2.3-GFP and DMP1-GFP pulps were processed for flow-cytometric sorting (FACS) analysis at day 7 by mild $0.05 \%$ trypsin/EDTA (Invitrogen) digestion followed by centrifugation at $4^{\circ} \mathrm{C}$. Cells were then resuspended in $300-400 \mu \mathrm{l}$ of the staining medium $(1 \times$ HBSS, $2 \%$ FBS and $10 \mathrm{mM}$ HEPES in distilled $\mathrm{H}_{2} \mathrm{O}, \mathrm{pH}$ 7.2) containing $1.0 \mu \mathrm{g} / \mathrm{ml}$ propidium iodide, and strained through a $70-\mu \mathrm{m}$ strainer to obtain single-cell suspension. Approximately $50,000-100,000$ cells/sample were collected by a $\mathrm{BD}^{\mathrm{TM}}$ LSR-II FACS cytometer (BD Biosciences, San Jose, Calif., USA) using a blue laser (excitation $488 \mathrm{~nm}$ at $20 \mathrm{~mW}$; collected emission at 515-545 nm). Percentages of GFP-positive and GFP-negative cells were determined using BD FACSDiva ${ }^{\mathrm{TM}} 6.2$ software. Pulp cells from nontransgenic littermates served as a negative control for GFP expression in all experiments.

For FACS sorting, pulp cells from 2.3-GFP pulps were grown under control culture conditions for 7 days. At day 7, cells were detached with $0.05 \%$ trypsin/EDTA (Invitrogen) followed by centrifugation at $4^{\circ} \mathrm{C}$. Cells were then resuspended in $300-400 \mu \mathrm{l}$ of the staining medium containing $1.0 \mu \mathrm{g} / \mathrm{ml}$ propidium iodide, and strained through a $70-\mu \mathrm{m}$ strainer. FACS based on GFP expression was performed on $2.5 \times 10^{6}$ cells $/ \mathrm{ml}$ by UCHC FACS facility using a BD FACSAria ${ }^{\mathrm{TM}}$ II cell sorter $(130 \mu \mathrm{m}$ nozzle at 12 PSI; BD Biosciences). GFP was excited at $488 \mathrm{~nm}$ with an argon laser and a
550/30 emission filter was utilized. Upon separation, reanalysis confirmed that the purity of isolated 2.3-GFP-positive and 2.3-GFP-negative populations was higher than 98\%. Live GFPpositive and GFP-negative cells were collected into DMEM with $20 \%$ FBS, recounted and replated at the same density as the primary cultures $\left(8.75 \times 10^{4}\right.$ cells $\left./ \mathrm{cm}^{2}\right)$. Cultures were treated with VH or FGF2 (20 ng/ml) between days 3 and 14 and processed for various analyses as described for unsorted cultures.

\section{Statistical Analysis}

Statistical analysis of the data was performed by GraphPad Prism 6 software using one-way ANOVA analysis with the Bonferroni multiple comparison post hoc test or the unpaired two-tailed Student $t$ test. Values in all experiments represented mean \pm SEM of at least 3 independent experiments, and $\mathrm{p} \leq 0.05$ was considered statistically significant.

\section{Results}

\section{Effects of FGF2 on Mineralization and Dentinogenesis} in Primary Dental Pulp Cultures

Previous studies in our laboratory showed that when placed in primary culture, progenitor/stem cells in the pulp from unerupted molars proliferated rapidly and reached confluence around day 7 (the proliferation phase of in vitro growth). Following the addition of the mineralization-inducing medium at day 7 , these cells underwent differentiation and gave rise to an extensive amount of mineralized matrix (the differentiation/mineralization phase of in vitro growth). The first sign of mineralization appeared around day 10 with significant increases in the extent of mineralization thereafter. At day 21, almost the entire culture dish was covered with a sheet of mineralized tissue [Balic et al., 2010a].

Using this well-characterized dental pulp culture system, we examined the effects of FGF2 on mineralization and dentinogenesis. In these experiments, primary pulp cultures were exposed to VH (control) or FGF2 between days 3 and 21 (during both proliferation and differentiation/mineralization phases of in vitro growth).

$\mathrm{XO}$ and von Kossa staining showed marked and concentration-dependent decreases in the extent of mineralization in FGF2-treated cultures compared to controls (fig. 1a, b). qPCR analysis also showed concentration-dependent changes in FGF2-treated cultures compared to controls (fig. 1c). FGF2-treated cultures showed increases in the levels of expression of all markers at day 7 followed by decreases between days 10 and 21 compared to controls. The most marked increases at day 7 in FGF2-treated cultures were in the expression of $D m p 1$ (up to approx. 310 -fold) followed by increases in the expression of $D s p p$, 

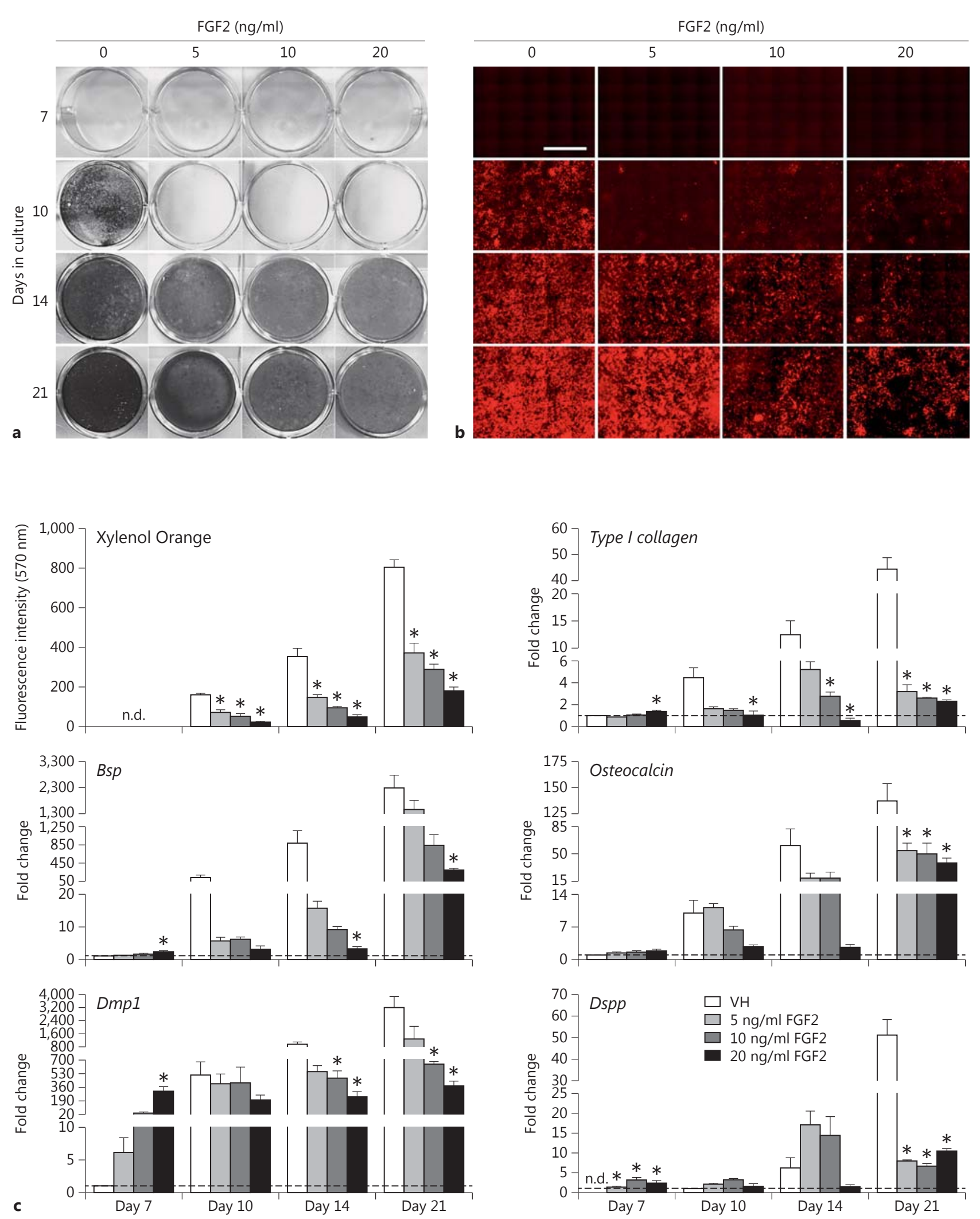

(For legend see next page.)

Stage-Specific Effects of FGF2 on Pulp Cells
Cells Tissues Organs 2014;199:311-328 DOI: $10.1159 / 000371343$ 
Table 1. Effects of FGF2 on the proliferation of dental pulp cells (WST-1 assay)

\begin{tabular}{llll}
\hline $\begin{array}{l}\text { Time point after } \\
\text { treatment, } \mathrm{h}\end{array}$ & $\mathrm{VH}$ & FGF2 & Fold change $^{\mathrm{a}}$ \\
\hline 24 & $0.56 \pm 0.01$ & $0.62 \pm 0.01^{*}$ & $\sim 1.11$ \\
48 & $0.82 \pm 0.05$ & $1.04 \pm 0.07^{*}$ & $\sim 1.27$ \\
72 & $0.77 \pm 0.03$ & $1.18 \pm 0.03^{*}$ & $\sim 1.53$ \\
96 & $0.61 \pm 0.03$ & $1.00 \pm 0.05^{*}$ & $\sim 1.64$ \\
\hline
\end{tabular}

Cultures were established from 5- to 7-day-old nontransgenic mice and treated with $\mathrm{VH}$ or $20 \mathrm{ng} / \mathrm{ml} \mathrm{FGF2}$ starting on day 3 $(0 \mathrm{~h})$. They were processed for the WST-1 proliferation assay at 24, 48, 72 and $96 \mathrm{~h}$ after FGF2 treatment (see Materials and Methods). Results represent the mean \pm SEM of absorbance in at least 3 independent experiments. ${ }^{*} \mathrm{p} \leq 0.05$, relative to control at each time point.

a The FGF2 value divided by the control value for each time point.

Bsp and osteocalcin (approx. 2-fold). These observations revealed both the stimulatory and inhibitory effects of FGF2 on the expression of markers of mineralization and dentinogenesis.

\section{Effects of FGF2 on Cell Proliferation in Primary \\ Dental Pulp Cultures}

To examine if the increases in the expression of various markers of dentinogenesis in FGF2-treated cultures at day 7 were related to increases in cell numbers, the effects of FGF2 on cell proliferation in the whole culture were examined by the WST-1 assay 24-96 h after exposure to FGF2 (on days 4-7 of the culture). Cell proliferation in

Fig. 1. Concentration-dependent effects of FGF2 on mineralization and the expression of markers of mineralization and dentinogenesis in primary dental pulp cultures. a Representative images of von Kossa-stained dishes. b Representative composite of $5 \times$ scanned images of $\mathrm{XO}$-stained live cultures at various time points. The magnifications of all micrographs are identical. Scale bar $=$ $2 \mathrm{~mm}$. c Histogram showing the changes in the intensity of XO fluorescence (expressed as absolute values) and in the levels of expression of various markers of mineralization and dentinogenesis (expressed as relative values). Expression of all mRNAs except $D s p p$ is normalized to $\mathrm{VH}$ at day 7 , which is arbitrarily set to 1 (dashed line). The expression of Dspp is normalized to $\mathrm{VH}$ at day 10 , which is arbitrarily set to 1 (dashed line). Results represent the mean \pm SEM of at least 3 independent experiments. Note the increases in the expression of markers of mineralization and dentinogenesis at day 7 followed by decreases in their expression at days 10-21 in FGF2-treated cultures compared to controls. * $\mathrm{p} \leq$ 0.05 , relative to $\mathrm{VH}$ at each time point. n.d. $=$ Not detected. the control and FGF2-treated cultures peaked at around 48-72 $\mathrm{h}$ and declined at $96 \mathrm{~h}$. FGF2-treated cultures showed an increase in proliferation of up to approximately 1.6-fold at 24-96 h compared to controls (table 1). These observations showed that FGF2 increased cell proliferation.

\section{Effects of FGF2 on Expression of Various GFP}

Reporter Transgenes in Primary Dental Pulp Cultures

Next, we examined the effects of FGF2 on pulp cultures from various GFP reporter transgenic mice. We used the expression of 2.3-GFP and 3.6-GFP transgenes as markers for cells at the early stages of odontoblast differentiation (polarizing odontoblasts that lack expression of Dmp1 and Dspp) [Balic et al., 2010b]. DMP1-GFP and DSPP-Cerulean transgenes were used as markers for cells at the later stages of odontoblast differentiation (functional and fully differentiated odontoblasts) [Balic and Mina, 2011; unpubl. data].

Epifluorescence analyses of live cultures at day 7 showed slight increases in the intensity of 2.3-GFP and 3.6-GFP transgenes and marked increases (approx. 38fold) in the intensity of the DMP1-GFP transgene (fig. 2 and data not shown) in FGF2-treated cultures compared to the respective controls. These increases in FGF2-treated cultures were followed by marked decreases in the intensity of the expression of all transgenes compared to the respective controls between days 10 and 14 (fig. 2 and data not shown). The percentage of DSPP-Cerulean-positive odontoblasts in FGF2-treated cultures at day 14 was less than half of that in the control cultures (control: 6.50 $\pm 0.18 \%$; FGF2-treated: $3.00 \pm 0.15 \%$; approx. 2.3 -fold; fig. 2a).

To determine if increases in the intensity of these transgenes in FGF2-treated cultures at day 7 were related to increases in the number of the GFP-positive cells, FACS analysis was performed at day 7 (table 2). FGF2treated cultures displayed slight but not significant increases in the percentages of the 2.3-GFP-positive and 3.6-GFP-positive populations compared to the respective controls (table 2). Pulp cultures from DMP1-GFP transgenic animals showed marked increases in the percentage of DMP1-GFP-positive cells compared to controls (table 2). Immunocytochemical analysis of pulp cultures from DSPP-Cerulean mice at day 7 also showed marked increases in the percentage of DSPP-Cerulean-positive odontoblasts in FGF2-treated cultures compared to controls (table 2). 


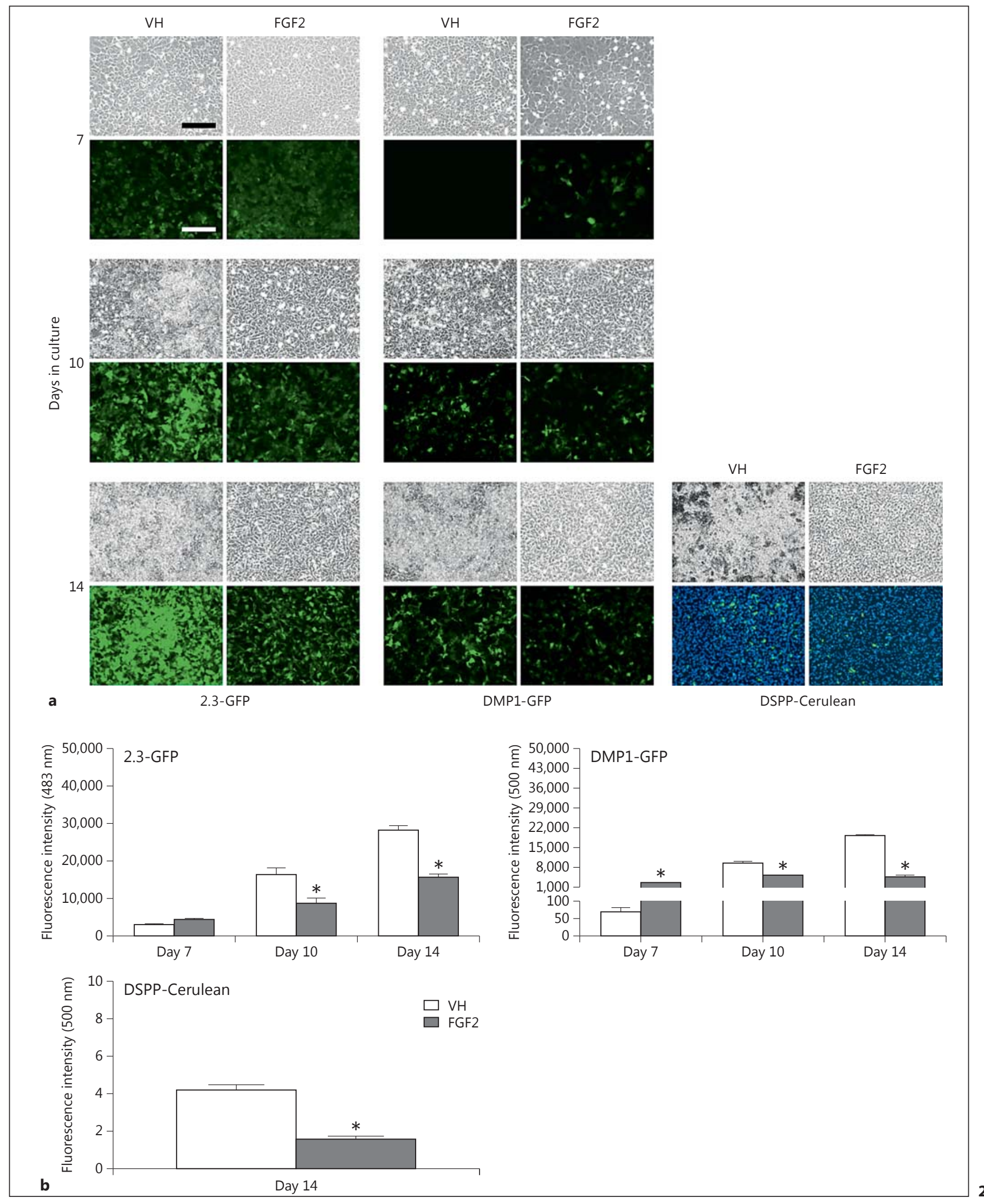

(For legend see next page.)

Stage-Specific Effects of FGF2 on Pulp Cells
Cells Tissues Organs 2014;199:311-328 DOI: $10.1159 / 000371343$ 
Table 2. Effects of FGF2 on the percentage of GFP-positive cells in primary dental pulp cultures

\begin{tabular}{|c|c|c|c|c|}
\hline & \multicolumn{2}{|l|}{ Controls } & \multicolumn{2}{|l|}{ FGF2-treated cells } \\
\hline & $\begin{array}{l}\% \text { GFP-negative } \\
\text { cells }\end{array}$ & $\begin{array}{l}\% \text { GFP-positive } \\
\text { cells }\end{array}$ & $\begin{array}{l}\% \text { GFP-negative } \\
\text { cells }\end{array}$ & $\begin{array}{l}\% \text { GFP-positive } \\
\text { cells }\end{array}$ \\
\hline 2.3-GFP & $15.10 \pm 0.80$ & $84.90 \pm 0.77$ & $12.57 \pm 0.64$ & $87.43 \pm 0.66$ \\
\hline 3.6-GFP & $17.31 \pm 0.18$ & $82.69 \pm 0.14$ & $15.62 \pm 0.35$ & $84.38 \pm 0.40$ \\
\hline DMP1-GFP & $94.60 \pm 0.27$ & $5.40 \pm 0.27$ & $64.60 \pm 2.98^{*}$ & $35.40 \pm 2.98^{*}$ \\
\hline DSPP-Cerulean & n.d. & $0.11 \pm 0.05$ & n.d. & $1.91 \pm 0.25^{*}$ \\
\hline
\end{tabular}

Cultures were treated with VH or $20 \mathrm{ng} / \mathrm{ml} \mathrm{FGF2} \mathrm{starting} \mathrm{at} \mathrm{day} \mathrm{3.} \mathrm{They} \mathrm{were} \mathrm{subjected} \mathrm{to} \mathrm{GFP-based} \mathrm{FACS}$ analysis and immunocytochemistry for DSPP-Cerulean at day 7. Results represent mean \pm SEM of at least 3 independent experiments. n.d. $=$ Not detected.

$* \mathrm{p} \leq 0.05$, relative to control at each time point.

Effects of FGF2 on FACS-Sorted 2.3-GFP-Positive and 2.3-GFP-Negative Populations

The presence of a mixture of GFP-positive and GFPnegative populations made it difficult to study the effects of FGF2 on activation of these transgenes during proliferation and mineralization/dentinogenesis. Therefore, as the next step, we studied the effects of FGF2 on FACSsorted populations. Our previous observations showed that FACS-sorted 2.3-GFP-positive and 2.3-GFP-negative populations represented proliferative cells enriched in polarizing odontoblasts and undifferentiated progenitors, respectively [Balic et al., 2010b]. Based on these observations, we examined the effects of FGF2 on relatively homogeneous populations of FACS-sorted 2.3-GFP-positive and 2.3-GFP-negative cells ( $\geq 98 \%$ purity of isolated populations; online suppl. fig. 1).

Fig. 2. Effects of FGF2 on the expression of GFP transgenes in primary dental pulp cultures. a Each panel represents images of the same areas in cultures from transgenic animals at different time points analyzed under bright-field and epifluorescent light using filters for GFPemd (for detection of the 2.3-GFP transgene) or GFPtpz (for detection of the DMP1-GFP transgene). The magnifications of all micrographs are identical. Scale bar $=200 \mu \mathrm{m}$. b Histograms showing changes in the intensity of 2.3-GFP, DMP1GFP and DSPP-Cerulean transgene expression in VH- and FGF2treated cultures. For detection of DSPP-Cerulean, cells were processed for immunocytochemistry using anti-GFP antibody at day 14 and epifluorescent image represents Hoechst/GFP overlay. Results are expressed as absolute values and represent the mean \pm SEM of at least 3 independent experiments. Note the marked increase in the intensity of DMP1-GFP at day 7 and the decreases in the intensity of 2.3-GFP and DMP1-GFP at days 10-14 in the FGF2-treated cultures compared to the controls. ${ }^{*} \mathrm{p} \leq 0.05$, relative to $\mathrm{VH}$ at each time point.
In cultures established from the 2.3-GFP-positive population, GFP expression was detected initially and was maintained throughout the entire culture period; the first sign of mineralization was around day 10 with significant increases thereafter (fig. 3a). In these cultures, low levels of Dmp1 and Dspp were detected around days 7 and 10, respectively. The expression of markers of mineralization and dentinogenesis increased with more advanced stages of differentiation in vitro (fig. $3 b$ ).

In cultures established from the 2.3-GFP-negative population, GFP was not detected initially, but appeared at day 7 in a few isolated cuboidal cells and increased thereafter (fig. 3a). In these cultures, low levels of Dmp1 were detected at day 7 , and mineralization and expression of Dspp were detected only at day 14 (fig. 3). The delayed expression of GFP and the delayed appearance of XO-stained mineralized nodules together with the lack of expression of $D s p p$ at days 7 and 10 in cultures from the 2.3-GFP-negative population confirmed that, in comparison to the 2.3-GFP-positive population, this population was enriched in cells at earlier stages of differentiation.

FGF2-treated cultures showed marked decreases in the intensity of GFP expression and the extent of mineralization compared to the respective controls. However, in the 2.3-GFP-positive cultures, FGF2 increased the expression of Dmp1 and Dspp at day 7, followed by decreases at days 10 and 14 compared to controls. In the 2.3-GFPnegative population, FGF2 increased the levels of Dmp1 at days 7 and 10 followed by decreases at day 14 compared to controls; in the 2.3-GFP-negative population, expression of Dspp was detected only at day 14 and at lower levels in FGF2-treated cultures compared to controls (fig. 3b). 


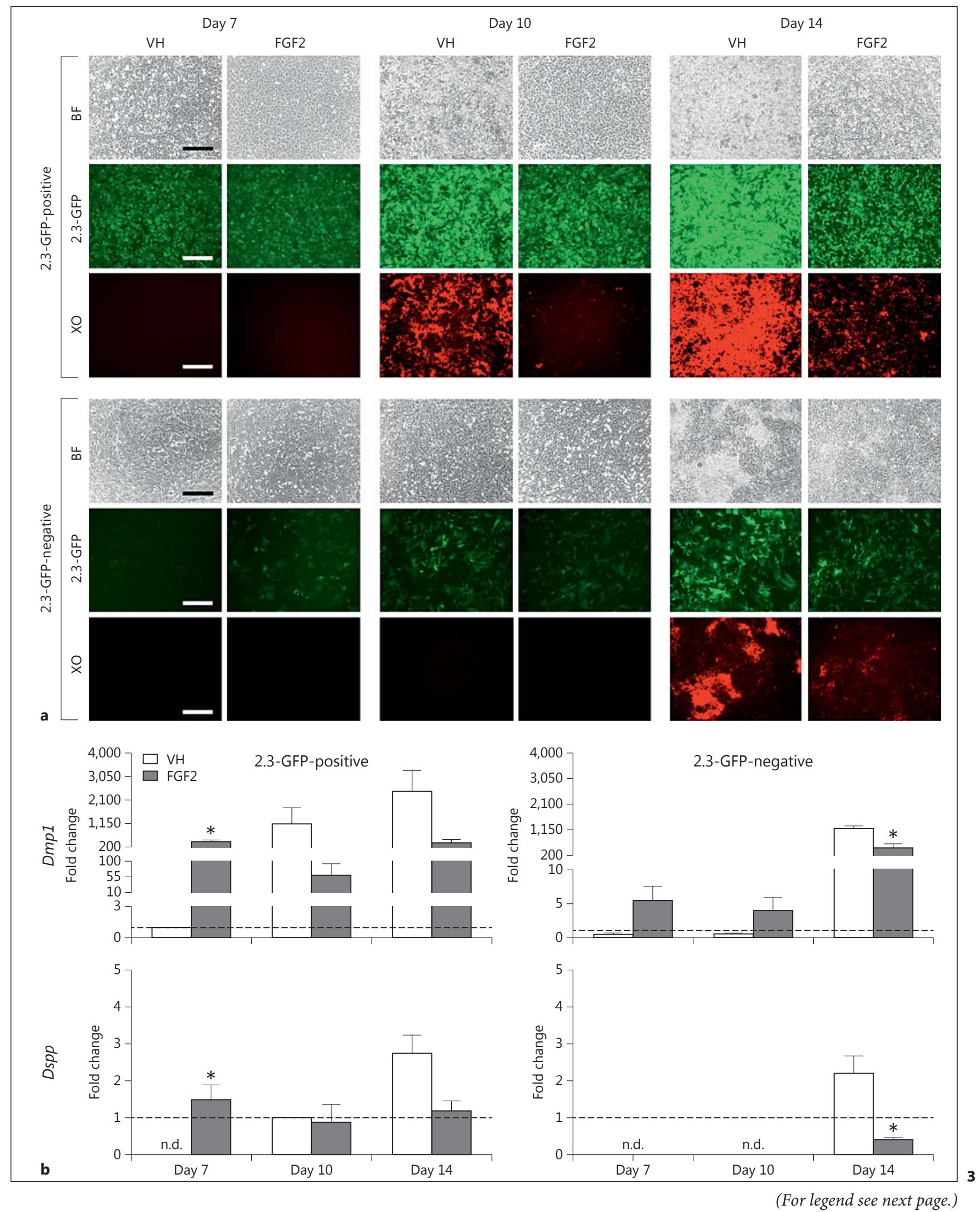

Stage-Specific Effects of FGF2 on Pulp Cells

Cells Tissues Organs 2014;199:311-328 DOI: $10.1159 / 000371343$ 
FGF2 Inhibited the Progression of Cells into the Final Stage of Differentiation

Despite decreases at days 10 and 14 compared to the respective controls, the intensity of 2.3-GFP and DMP1GFP in FGF2-treated cultures remained relatively unchanged (fig. 2). These observations suggested that FGF2 did not dedifferentiate cells and maintained healthy numbers of 2.3-GFP-positive and DMP1-GFP-positive cells, in which further differentiation into mature odontoblasts was inhibited. To test this possibility, we examined the effects of withdrawal of FGF2 on the differentiation of pulp cells. In these experiments, dental pulp cells were exposed to FGF2 between days 3 and 14, and were then grown in control medium (without FGF2) for an additional 7 days. The effects of withdrawal of FGF2 on the extent of mineralization and dentinogenesis in these cultures were compared to the control cultures (not exposed to FGF2) and cultures that were continuously exposed to FGF2 between days 3 and 21 .

Withdrawal of FGF2 for 7 days allowed almost complete recovery of mineralization and the expression of markers of mineralization and dentinogenesis (fig. 4). The intensity of $\mathrm{XO}$ staining at day 21 in these cultures was similar to that in the control cultures (fig. 4b). The levels of expression of type I collagen, Bsp and osteocalcin in these cultures at day 21 were higher than those in the cultures continuously exposed to FGF2, but did not reach control levels. On the other hand, the levels of Dmp1 in these cultures were similar to those in the controls, and the levels of Dspp were higher (approx. 3.2-fold) than those in the controls.

Epifluorescence analyses of cultures from various transgenic animals showed that 7 days after the withdrawal of FGF2, the intensity of the expression of 2.3-GFP and 3.6-GFP transgenes reached that in the respective

Fig. 3. Effects of FGF2 on the mineralization and expression of the 2.3-GFP transgene in FACS-sorted 2.3-GFP-positive and 2.3-GFPnegative populations. Primary pulp cultures from the 2.3-GFP transgenic mice were grown under control culture conditions and processed for FACS to separate relatively homogeneous 2.3-GFPpositive and 2.3-GFP-negative populations. The FACS-sorted populations were plated (day 0) and exposed to $\mathrm{VH}$ or $20 \mathrm{ng} / \mathrm{ml}$ FGF2 between days 3 and 14. a Each panel represents images of the same areas in cultures at different time points analyzed under bright-field (BF) and epifluorescent light using appropriate filters for the detection of GFP and XO. The magnifications of all micrographs are identical. Scale bar $=200 \mu \mathrm{m}$. Note the decreases in mineralization in both populations in response to FGF2. b Histograms showing the changes in the expression of Dmp1 and Dspp in both populations. Expression levels of $D m p 1$ in both popula- control cultures (fig. 5 and data not shown). Interestingly, the intensity of DMP1-GFP and DSPP-Cerulean transgenes and the percentage of DSPP-Cerulean-positive odontoblasts (control: $6.88 \pm 0.28 \%$. FGF2-treated: $8.39 \pm$ $0.21 \% ; 1.22$-fold) were higher than those in the respective controls (fig. 5).

\section{Effects of FGF2 on Primary BMSC Cultures}

Our previous studies showed that primary dental pulp cultures from unerupted molars contained progenitors capable of giving rise to both osteoblasts and odontoblasts [Balic et al., 2010a]. This makes it difficult to distinguish the effects of FGF2 on cells of osteogenic versus dentinogenic lineages.

To distinguish between the effects of FGF2 on the cells of these lineages, we examined the effects of FGF2 on BMSC cultures, as they do not contain odontoprogenitors and are used routinely to examine mineralization and osteoblast differentiation in vitro. Previous studies have also shown that in the osteoblast lineage, 3.6-GFP is activated in preosteoblasts, 2.3-GFP in mature osteoblasts and DMP1-GFP in late osteoblasts and osteocytes [Kalajzic et al., 2002, 2004].

Exposure of BMSCs to FGF2 between days 3 and 14 completely inhibited mineralization (fig. 6a) and led to marked decreases in the expression of markers in the early and late stages of osteoblast differentiation at day 7 , except for Dmp1 which was transiently increased (approx. 35-fold) compared to controls (fig. 6c). At days 10 and 14, expression of all these markers was markedly reduced in FGF2-treated cultures compared to controls.

Analysis of BMSC cultures from various transgenic animals showed that FGF2 completely inhibited the expression of 2.3-GFP and 3.6-GFP transgenes at all time points compared to the respective controls. FGF2-treated

tions are normalized to the VH-treated 2.3-GFP-positive population at day 7, which is arbitrarily set to 1 (dashed line). Expression levels of Dspp in both populations are normalized to the VH-treated 2.3-GFP-positive population at day 10, which is arbitrarily set to 1 (dashed line). Results represent mean \pm SEM of at least 3 independent experiments. In the 2.3-GFP-positive population, the FGF2-treated cultures displayed increases in the levels of Dmp1 and $D s p p$ at day 7 followed by decreases in the levels of their expression at days 10-14 compared to controls, and in the 2.3-GFPnegative population, they showed increases in the levels of $D m p 1$ at day 7 compared to controls. At later time points, these cultures displayed decreases in the expression of Dmp1 and Dspp compared to controls. n.d. $=$ Not detected. ${ }^{*} \mathrm{p} \leq 0.05$, relative to $\mathrm{VH}$ at each time point. 


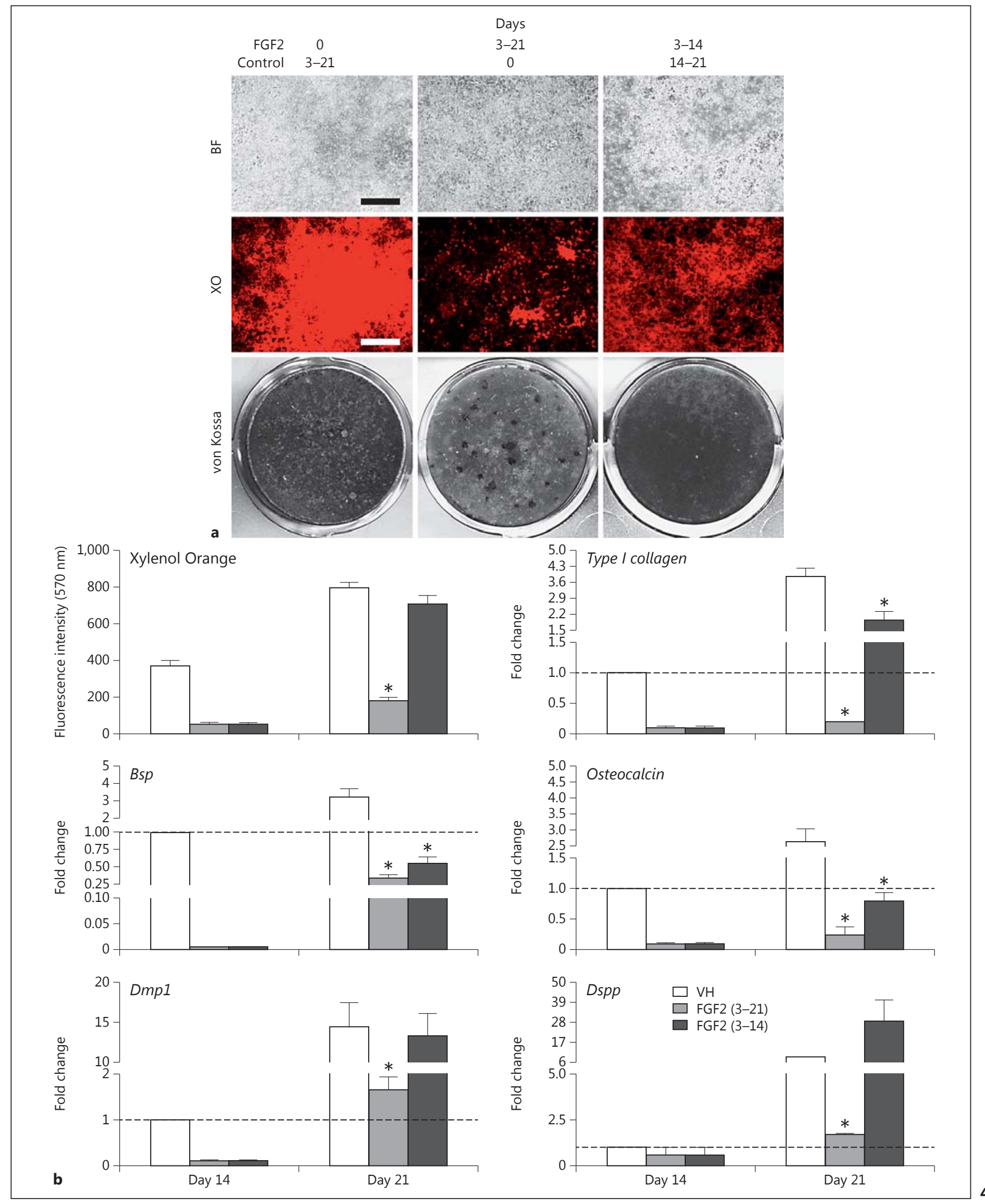

(For legend see next page.) 
BMSC cultures from DMP1-GFP animals showed a few DMP1-GFP-positive cells at day 7 followed by complete inhibition of the expression of this transgene at days 10 and 14 compared to controls (fig. 6b). Expression of Dspp and DSPP-Cerulean transgene was not detected in control or FGF2-treated cultures at any time point (fig. 6c and data not shown).

\section{Discussion}

Members of the FGF family of growth factors including FGF2 play an essential role in various functions of dental pulp cells during reparative dentinogenesis, including the proliferation, migration, differentiation and self-renewal of dental pulp stem cells and progenitor cells [Nakao et al., 2004; He et al., 2008; Morito et al., 2009; Shimabukuro et al., 2009; Osathanon et al., 2011; Suzuki et al., 2011; Wu et al., 2012]. However, the effects of FGF2 on mineralization and dentinogenesis have remained controversial, as both inhibitory and stimulatory roles of FGF2 have been reported.

It has been shown that continuous exposure of primary dental pulp cultures and tooth organ cultures to FGF2 decrease the extent of mineralization and the expression of various markers of mineralization and dentinogenesis, including Dmp1 and Dspp [Tsuboi et al., 2003; He et al., 2008; Osathanon et al., 2011; Wu et al., 2012]. Inhibition of FGF2 signaling in tooth organ cultures by specific antisense oligonucleotides were found to increase Alp and Dspp expression [Tsuboi et al., 2003]. On the other hand, several studies have shown that FGF2 increases the expression of Dmpl and Dspp in primary pulp cultures [Nakao et al., 2004; Kim et al., 2010], immortalized human dental pulp cells [Kim et al., 2010] and E15 (cap stage) tooth organ cultures [Tsuboi et al., 2003].

Our study showed that the effects of FGF2 on the differentiation of pulp cells were stage-specific and depended on the stage of maturity of cells. Our results provide

Fig. 4. Effects of withdrawal of FGF2 on mineralization and the expression of markers of mineralization and dentinogenesis in primary dental pulp cultures. Cultures were treated with VH or $20 \mathrm{ng} /$ $\mathrm{ml} \mathrm{FGF2}$ between days 3 and 14. At day 14, FGF2 was withdrawn and cells were grown for an additional 7 days in the medium without FGF2. a Images of the same areas in cultures at day 21 analyzed under bright-field (BF, upper row) and epifluorescent light using TRITC Red filter for the detection of XO staining (middle row). The lower row shows representative von Kossa-stained dishes. The strong support for FGF2 stimulating/promoting the differentiation of early progenitors into functional odontoblasts in the odontoblast lineage (fig. 7). The exposure of pulp cells to FGF2 during the proliferation phase of in vitro growth increased the levels of the expression of all markers of mineralization and dentinogenesis, including a marked increase in Dmpl expression and the intensity of the DMP1-GFP transgene, shown to be activated in functional odontoblasts [Balic and Mina, 2011]. Furthermore, our studies on FACS-sorted populations show that FGF2 stimulated the expression of Dmp1 at day 7 in both undifferentiated progenitors (2.3-GFP-negative) and in cells at the early stages of differentiation (polarizing odontoblasts, 2.3-GFP-positive). FGF2 stimulated Dspp expression only in the 2.3-GFP-positive population.

Our results also show that, despite these early stimulatory effects, additional exposure of pulp cells to FGF2 reduced mineralization, expression of $D m p 1$ and $D s p p$, all transgenes and the number of DSPP-Cerulean-positive odontoblasts compared to controls (fig. 7). These observations together with those on FACS-sorted populations suggest that in the odontoblast lineage, FGF2 inhibits the differentiation of functional odontoblasts into fully differentiated odontoblasts. The rapid and almost complete recovery of mineralization and the expression of markers of dentinogenesis and various GFP transgenes 7 days after withdrawal of FGF2 suggest that the inhibitory effects of FGF2 on mineralization and dentinogenesis are primarily related to its negative effects on the final stages of cell differentiation.

Taken together, these results show the stage-specific effects of FGF2 on the differentiation of odontoblast lineage cells and suggest a positive role of FGF2 in the formation of functional odontoblasts and a negative role in the further differentiation of these cells. Additional experiments are in progress to examine the underlying mechanisms mediating the stimulatory and inhibitory effects of FGF2 on pulp cells. These observations provide an insight into conflicting results for positive and nega-

magnifications of all micrographs are identical. Scale bar $=200 \mu \mathrm{m}$. b Histograms showing the changes in the intensity of XO staining (relative values). Levels of expression of all mRNAs were normalized to those of $\mathrm{VH}$ at day 14, which is arbitrarily set to 1 (dashed line). Results represent the mean \pm SEM of values from at least 3 independent experiments. Note the almost complete recovery in the extent of mineralization and levels of expression of Dmp1 and $D s p p$ in the cultures after the withdrawal of FGF2 for 7 days. ${ }^{*} \mathrm{p} \leq$ 0.05 , relative to $\mathrm{VH}$ at each time point. 
tive effects of FGF2 on mineralization and dentinogenesis.

It is well documented that FGF signaling produces diverse biological responses in various cell types. The mechanisms of specific cellular responses to FGF signaling are dependent on many factors, including cell type, the expression of specific ligands and receptors, the signal transduction pathways utilized and the transcriptional regulation of tissue-specific genes [Dailey et al., 2005]. Moreover, studies on bone show that the response to FGF signaling in a specific cell type is also stage-specific. FGF signaling stimulates the proliferation of immature osteoblasts but inhibits mineralization and increased apoptosis in more differentiated cells [Mansukhani et al., 2000; Fakhry et al., 2005; Eda et al., 2008; James et al., 2008; Xiao et al., 2013].

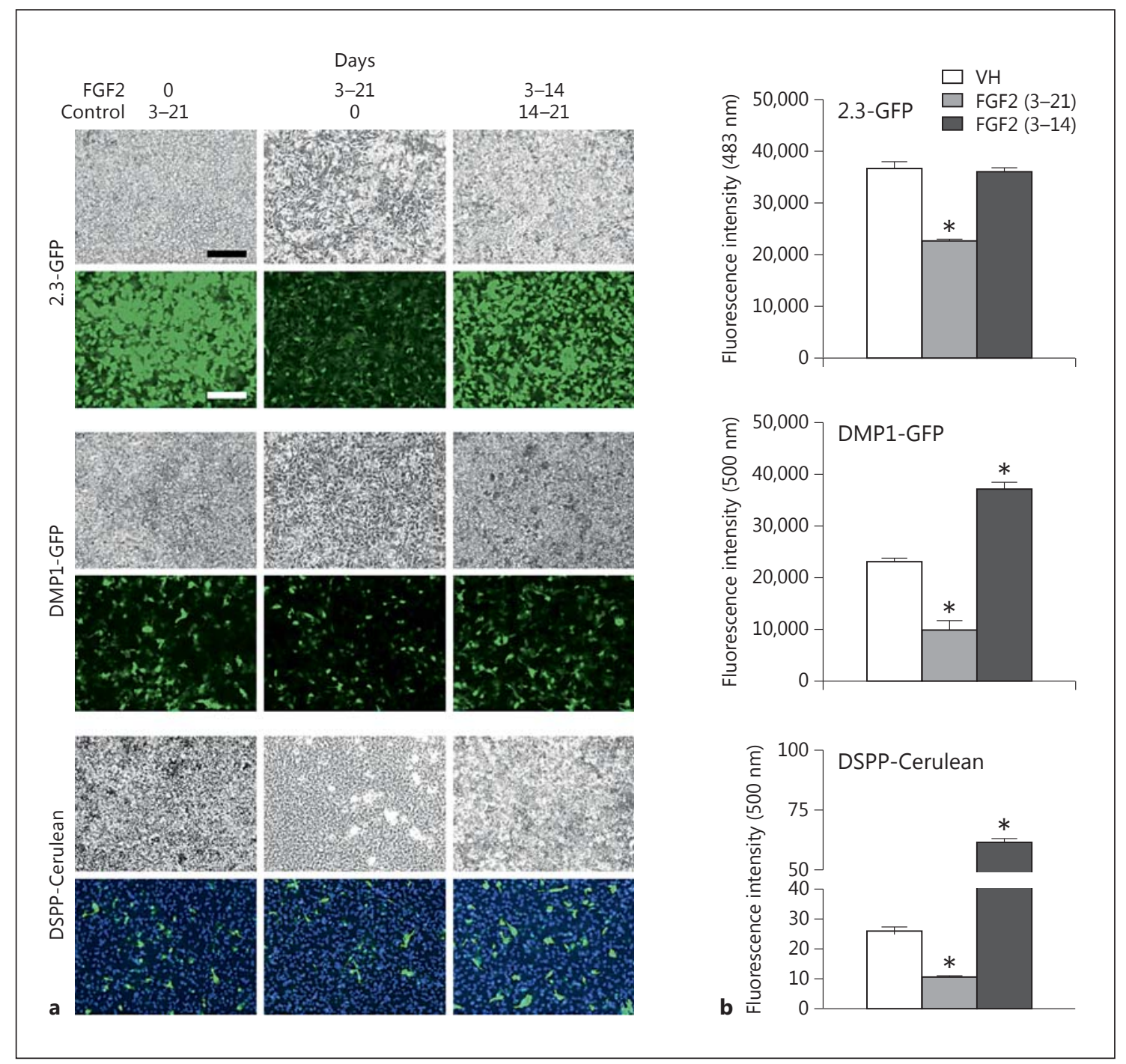

Fig. 5. Effects of the withdrawal of FGF2 on the expression of GFP transgenes in primary dental pulp cultures. a Representative images of the same areas in cultures analyzed under bright-field and epifluorescent light using appropriate filters at day 21. DSPP-Cerulean was detected with anti-GFP antibody by immunocytochemistry and examined using filter for GFPtpz. The epifluorescent image represents Hoechst/GFP overlay. The magnifications of all micrographs are identical. Scale bar $=200 \mu \mathrm{m}$. $\mathbf{b}$ Histograms showing the effects of withdrawal of FGF2 on the expression of various transgenes at day 21 . Results are expressed in absolute values and represent the mean \pm SEM of at least 3 independent experiments. Note that after withdrawal of FGF2 the intensity of the expression of 2.3-GFP reached that in control, and the intensity of DMP1-GFP and DSPP-Cerulean exceeded that in control. ${ }^{*} \mathrm{p} \leq$ 0.05 relative to $\mathrm{VH}$. 


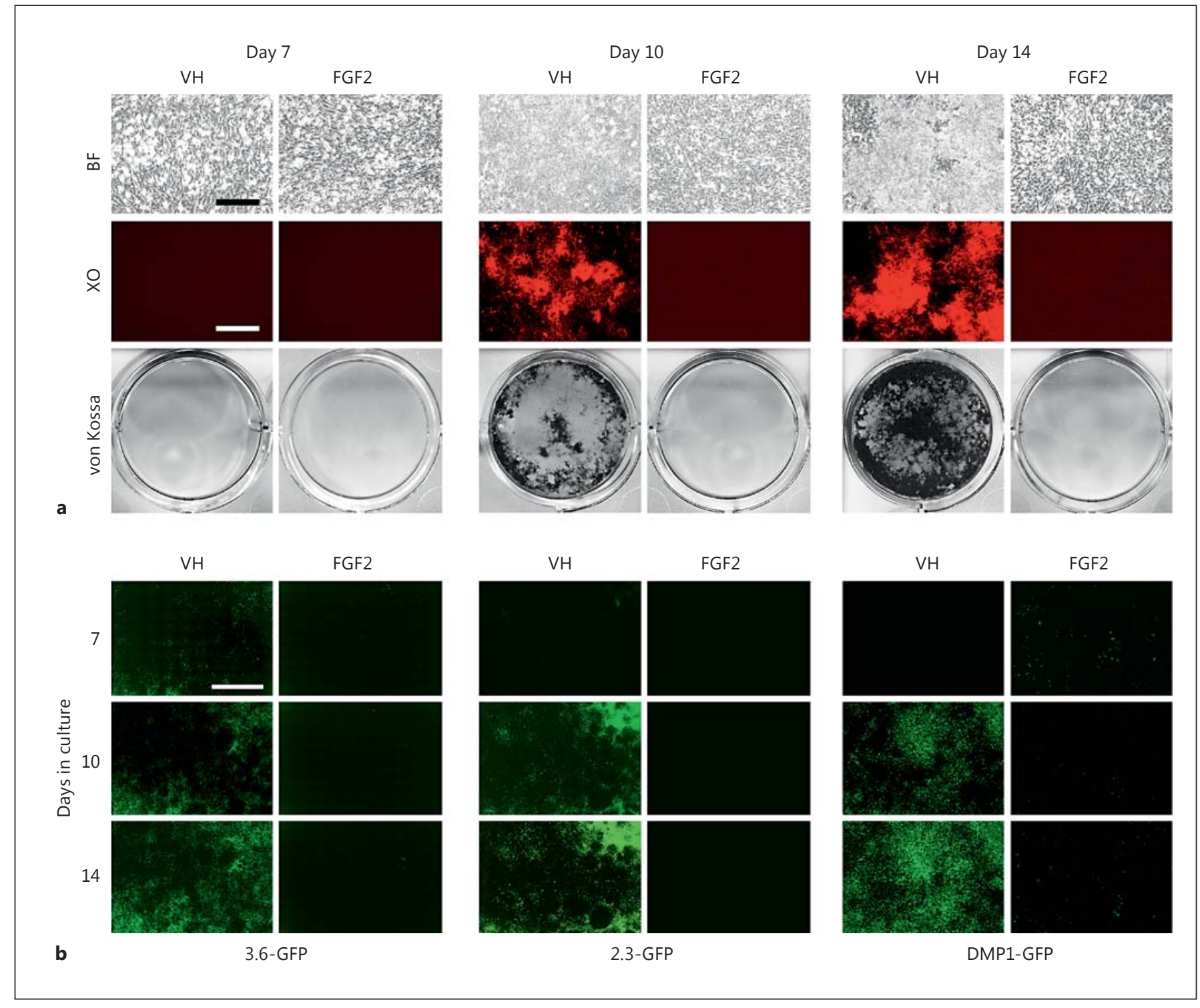

Fig. 6. Effects of FGF2 on mineralization, expression of transgenes and markers of mineralization in primary BMSC cultures. Primary BMSC cultures were treated with $\mathrm{VH}$ or $20 \mathrm{ng} / \mathrm{ml} \mathrm{FGF} 2$ between days 3 and 14. a Representative images of the same areas in cultures at different time points were analyzed under bright-field (BF, upper row) and epifluorescent light using TRITC Red filter for the detection of XO staining (middle row). The magnifications of all micrographs are identical. Scale bar $=200 \mu \mathrm{m}$. The lower row shows representative images of von Kossa-stained cultures. Note the lack of mineralization in FGF2-treated cultures compared to controls. b Representative composite of $5 \times$ scanned images of live cultures at various time points analyzed under epifluorescent light using filters for GFPtpz (for the detection of the 3.6-GFP and
DMP1-GFP transgenes) and GFPemd (for detection of 2.3-GFP transgene). The magnifications of all micrographs are identical. Scale bar $=2 \mathrm{~mm}$. Note the lack of expression of all transgenes in FGF2-treated cultures compared to controls. c Histograms showing the changes in the levels of expression of various markers of mineralization. Expression of all mRNAs is normalized to $\mathrm{VH}$ at day 7, which is arbitrarily set to 1 (dashed line). Results represent the mean \pm SEM of at least 3 independent experiments. n.d. $=$ Not detected. Note the marked increases in the levels of Dmp1 at day 7 and the marked decreases in the expression of all markers of mineralization at days 10 and 14 in the FGF2-treated cultures compared to the controls. ${ }^{*} \mathrm{p} \leq 0.05$, relative to $\mathrm{VH}$ at each time point. (For figure $6 c$ see next page.) 

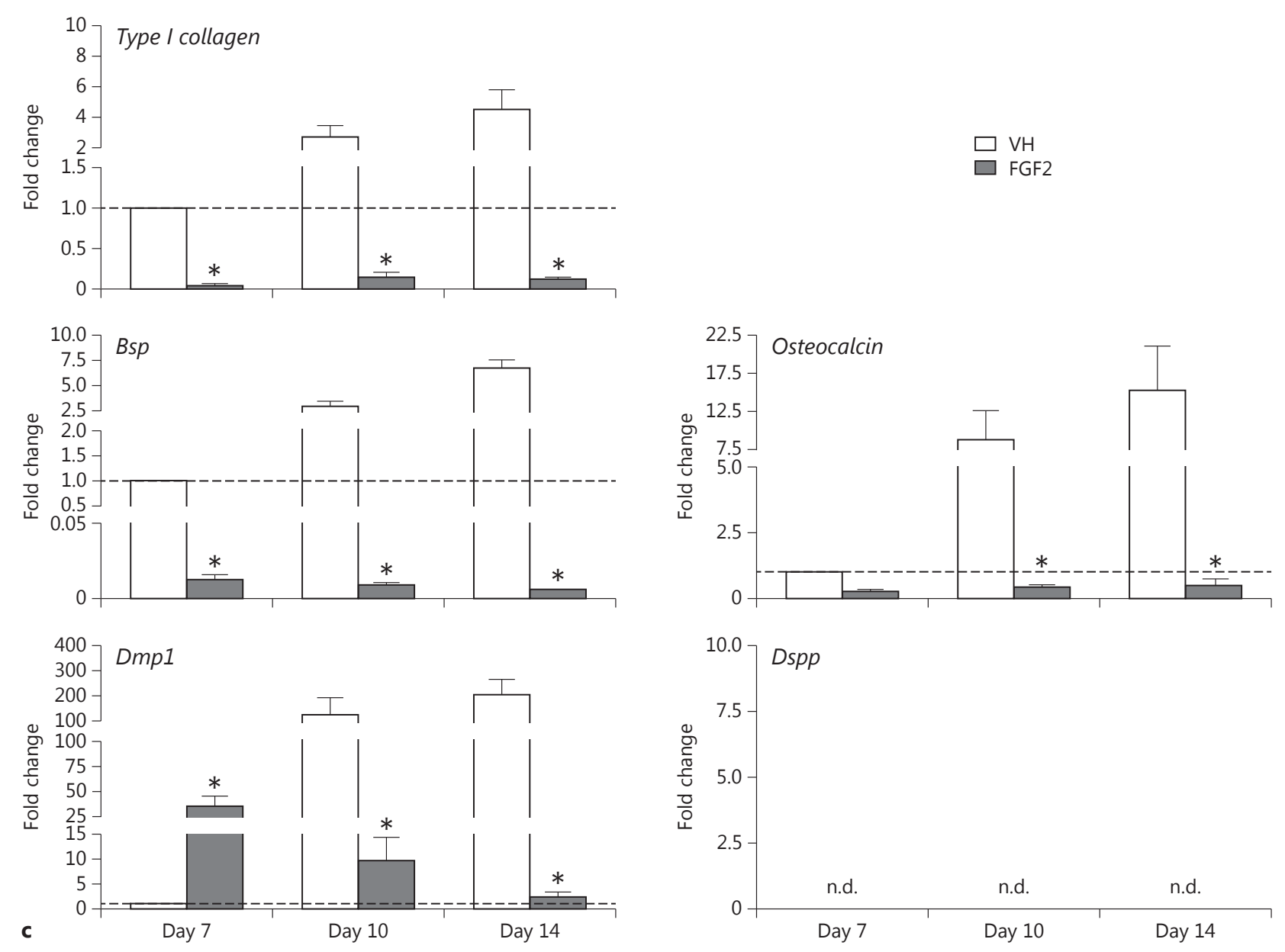

The Exposure of Primary BMSC Cultures to FGF2 Reveals Differences between the Effect of FGF2 on Osteoprogenitors and Odontoprogenitors

The formation of both bone- and dentin-like tissues in primary pulp cultures [Balic et al., 2010a] raises the possibility that some of the effects of FGF2 on dental pulp cultures may be related to its effects on cells of the osteoblast lineage. However, the differences between the early and later effects of FGF2 on BMSC and pulp cultures in our study suggest that in pulp cultures, it primarily affects cells of the odontoblast lineage.

Our results showed that continuous exposure of BMSCs to FGF2 completely inhibited mineralization and decreased the expression of markers of the early and late stages of osteoblast differentiation. This is consistent with previously reported studies [Kalajzic et al., 2003; Marie, 2012; Marie et al., 2012; Yamachika et al.,
2012]. The transient increase in Dmp1 in FGF2-treated cultures is also consistent with other studies that showed that exposure to FGF2 during the proliferation phase of in vitro growth resulted in rapid and marked increases in the expression of Dmp1 and other osteocyte-associated markers (E11, Cx43 and Phex) in osteoblast- and osteocyte-like cells (ROS17/2.8 and MC-4, MLO-Y4) and BMSC [Kyono et al., 2012; Nakayama et al., 2012]. Although the underlying mechanisms of the stimulatory effects of FGF2 on Dmp1 are not fully understood, the available evidence suggests the involvement of FGFR/MEK/Erk1/2 in this regulation [Kyono et al., 2012].

Furthermore, consistent with previous results [Kalajzic et al., 2003], our study showed that FGF2 completely inhibited the expression of the 3.6-GFP and 2.3-GFP transgenes in BMSC cultures, indicating that the inhibi- 


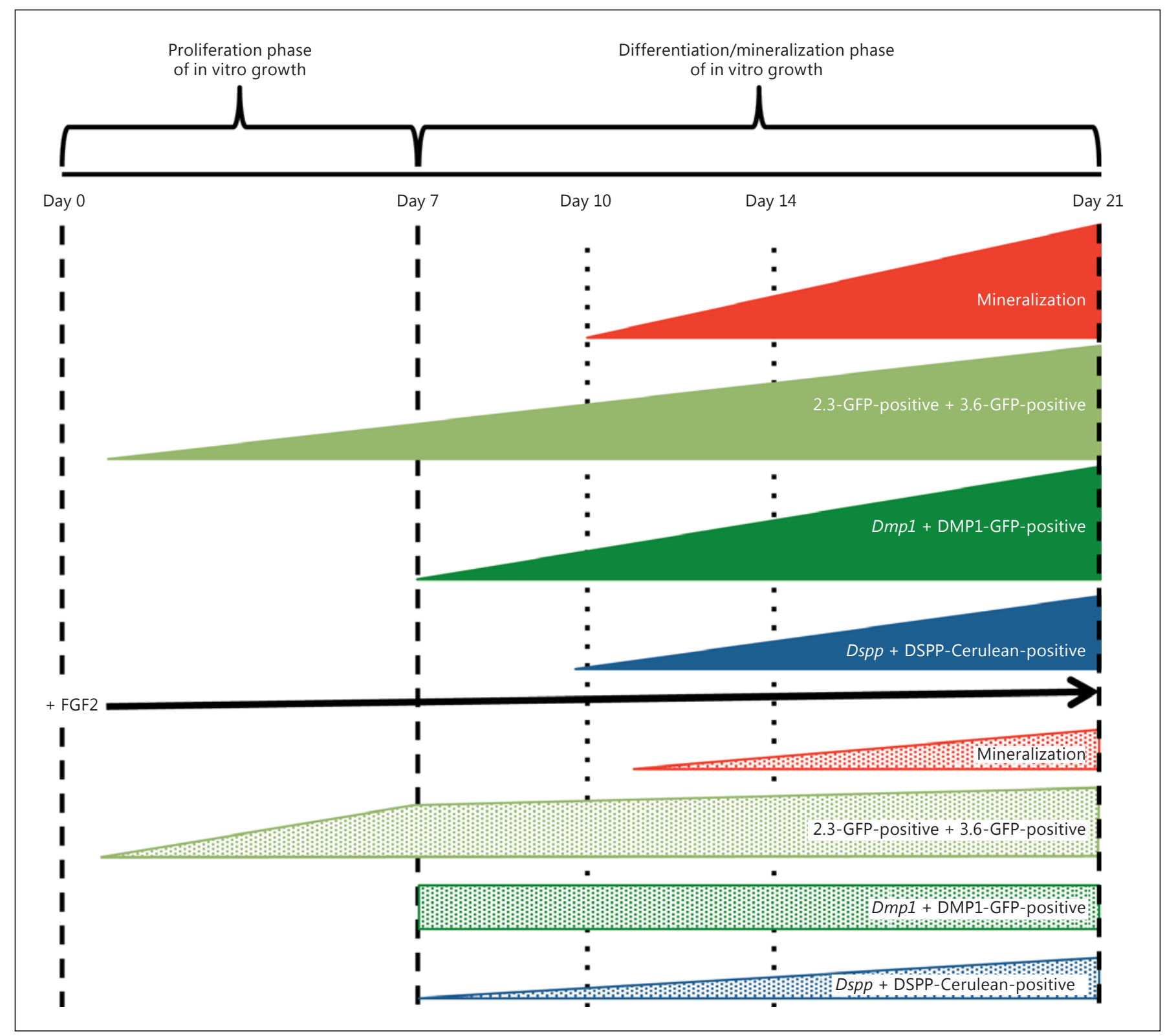

Fig. 7. Summary of changes in pulp cultures grown in the presence and absence of FGF2. During the proliferative phase of in vitro growth (i.e. the first 7 days), pulp cultures undergo proliferation and contain early progenitors. Following the addition of the mineralization-inducing medium at day 7 , these cells undergo differentiation and give rise to an extensive amount of mineralized matrix (the differentiation/mineralization phase of in vitro growth). The first sign of mineralization is around day 10 with significant increases in the extent of mineralization thereafter. In these cultures, Dmp1 and Dspp are expressed at low levels at day 7 and 10, respectively. DMP1-GFP-positive and DSPP-Cerulean-positive cells are detected at day 7 and 10, respectively, with increases there- after. The continuous exposure of pulp cultures to FGF2 resulted in decreases in the extent of mineralization. FGF2-treated cultures displayed increases in the levels of $D m p 1$, and the percentage of DMP1-GFP-positive cells at day 7 was followed by decreases between days 10 and 21 compared to controls. However, despite these decreases, the intensity of the DMP1-GFP transgene and the expression of Dmp1 in the FGF2-treated cultures between days 7 and 21 remained relatively unchanged. The FGF2-treated cultures also displayed increases in the level of $D s p p$ at day 7 , followed by a decrease in the levels of Dspp and the percentage of DSPP-Cerulean-positive cells between days 10 and 21 compared to the controls. 
tion of osteogenesis by FGF2 was mediated by blocking the onset of preosteoblast differentiation.

Our results showed that in the pulp cultures, FGF2 induced transient increases in the expression of all markers of mineralization and dentinogenesis at day 7 and reduced (but did not eliminate) the expression of the 3.6GFP and 2.3-GFP transgenes between days 10 and 21 .

In addition, the rapid and almost complete recovery of mineralization in the pulp cultures after the withdrawal of FGF2 in our study is different from that in the BMSC cultures. Upon withdrawal of FGF2 from BMSC cultures, full osteoblast differentiation and mineralization did not appear in vitro and was detected only after the subcutaneous implantation of FGF2-treated cells in SCID/Beige mice in vivo [Kalajzic et al., 2003].

These observations suggest significant differences in the response of odontoprogenitors and osteoprogenitors to FGF2 and/or differences in osteoprogenitors residing in the dental pulp as opposed to the bone marrow. Examining the differences in the activation of 2.3-GFP and DMP1-GFP in the cells of the osteogenic versus the dentinogenic lineage will facilitate a better understanding of such differences. Previous studies have showed that 2.3-
GFP and DMP1-GFP are activated in mature and late osteoblasts and osteocytes, respectively, i.e. in cell populations at relatively advanced stages of osteoblast differentiation [Kalajzic et al., 2002, 2004]. Our studies indicate that 2.3-GFP and DMP1-GFP are activated in polarizing and functional odontoblasts, respectively. These are cell populations at early and intermediate stages of odontoblast differentiation [Balic et al., 2010b; Balic and Mina, 2011].

\section{Acknowledgements}

We would like to thank all individuals who provided reagents, valuable input and technical assistance for this study, including Drs. David Rowe, Peter Maye and Anamaria Balic, Gloria Gronowicz, Mrs. Barbara Rodgers and members of the Molecular Core and Flow Cytometry Facilities at UCHC. This work was supported by grants No. R01-DE016689 and T90-DE022526 from the National Institute of Health.

\section{Disclosure Statement}

The authors declare no conflicts of interest.

\section{References}

Balic, A., H.L. Aguila, M.J. Caimano, V.P. Francone, M. Mina (2010a) Characterization of stem and progenitor cells in the dental pulp of erupted and unerupted murine molars. Bone 46: 1639-1651.

Balic, A., H.L. Aguila, M. Mina (2010b) Identification of cells at early and late stages of polarization during odontoblast differentiation using pOBCol3.6GFP and pOBCo12.3GFP transgenic mice. Bone 47: 948-958.

Balic, A., M. Mina (2011) Identification of secretory odontoblasts using DMP1-GFP transgenic mice. Bone 48: 927-937.

Braut, A., E.J. Kollar, M. Mina (2003) Analysis of the odontogenic and osteogenic potentials of dental pulp in vivo using a Colla1-2.3-GFP transgene. Int J Dev Biol 47: 281-292.

Cooper, P.R., Y. Takahashi, L.W. Graham, S. Simon, S. Imazato, A.J. Smith (2010) Inflammation-regeneration interplay in the dentinepulp complex. J Dentistry 38: 687-697.

Dailey, L., D. Ambrosetti, A. Mansukhani, C. Basilico (2005) Mechanisms underlying differential responses to FGF signaling. Cytokine Growth Factor Rev 16: 233-247.

-Eda, H., K. Aoki, K. Marumo, K. Fujii, K. Ohkawa (2008) FGF-2 signaling induces downregulation of TAZ protein in osteoblastic MC3T3E1 cells. Biochem Biophys Res Commun 366: 471-475.
Fakhry, A., C. Ratisoontorn, C. Vedhachalam, I. Salhab, E. Koyama, P. Leboy, M. Pacifici, R.E. Kirschner, H.D. Nah (2005) Effects of FGF2/-9 in calvarial bone cell cultures: differentiation stage-dependent mitogenic effect, inverse regulation of BMP-2 and noggin, and enhancement of osteogenic potential. Bone 36: 254-266.

Hatch, N.E. (2010) FGF signaling in craniofacial biological control and pathological craniofacial development. Crit Rev Eukaryot Gene Expr 20: 295-311.

He, H., J. Yu, Y. Liu, S. Lu, H. Liu, J. Shi, Y. Jin (2008) Effects of FGF2 and TGFbetal on the differentiation of human dental pulp stem cells in vitro. Cell Biol Int 32: 827-834.

Ishimatsu, H., C. Kitamura, T. Morotomi, Y. Tahata, T. Nishihara, K.K. Chen, M. Terashita (2009) Formation of dentinal bridge on surface of regenerated dental pulp in dentin defects by controlled release of fibroblast growth factor-2 from gelatin hydrogels. J Endod 35: 858-865.

James, A.W., Y. Xu, R. Wang, M.T. Longaker (2008) Proliferation, osteogenic differentiation, and FGF-2 modulation of posterofrontal/sagittal suture-derived mesenchymal cells in vitro. Plast Reconstr Surg 122: 53-63.
Kalajzic, I., A. Braut, D. Guo, X. Jiang, M.S. Kronenberg, M. Mina, M.A. Harris, S.E. Harris, D.W. Rowe (2004) Dentin matrix protein 1 expression during osteoblastic differentiation, generation of an osteocyte GFP-transgene. Bone 35: 74-82.

Kalajzic, I., Z. Kalajzic, M.M. Hurley, A.C. Lichtler, D.W. Rowe (2003) Stage-specific inhibition of osteoblast lineage differentiation by FGF2 and noggin. J Cell Biochem 88: 11681176.

Kalajzic, I., Z. Kalajzic, M. Kaliterna, G. Gronowicz, S.H. Clark, A.C. Lichtler, D. Rowe (2002) Use of type I collagen green fluorescent protein transgenes to identify subpopulations of cells at different stages of the osteoblast lineage. J Bone Miner Res 17: 15-25.

Kikuchi, N., C. Kitamura, T. Morotomi, Y. Inuyama, H. Ishimatsu (2007) Formation of dentin-like particles in dentin defects above exposed pulp by controlled release of fibroblast growth factor 2 from gelatin hydrogels. J Endod 33: 1198-1202.

Kim, J., J.C. Park, S.H. Kim, G.I. Im, B.S. Kim, J.B. Lee, E.Y. Choi, J.S. Song, K.S. Cho, C.S. Kim (2014) Treatment of FGF-2 on stem cells from inflamed dental pulp tissue from human deciduous teeth. Oral Dis 20: 191-204.
Stage-Specific Effects of FGF2 on Pulp Cells 
Kim, Y.S., K.S. Min, D.H. Jeong, J.H. Jang, H.W. Kim, E.C. Kim (2010) Effects of fibroblast growth factor- 2 on the expression and regulation of chemokines in human dental pulp cells. J Endod 36: 1824-1830.

Kuhn, L.T., Y. Liu, M. Advincula, Y.H. Wang, P. Maye, A.J. Goldberg (2010) A nondestructive method for evaluating in vitro osteoblast differentiation on biomaterials using osteoblastspecific fluorescence. Tissue Eng Part C Methods 16: 1357-1366.

Kyono, A., N. Avishai, Z. Ouyang, G.E. Landreth, S. Murakami (2012) FGF and ERK signaling coordinately regulate mineralization-related genes and play essential roles in osteocyte differentiation. J Bone Miner Metab 30: 19-30.

Li, C.Y., J. Prochazka, A.F. Goodwin, O.D. Klein (2014) Fibroblast growth factor signaling in mammalian tooth development. Odontology 102: 1-13.

Madan, A.K., B. Kramer (2005) Immunolocalization of fibroblast growth factor-2 (FGF-2) in the developing root and supporting structures of the murine tooth. J Mol Histol 36: 171-178.

Mansukhani, A., P. Bellosta, M. Sahni, C. Basilico (2000) Signaling by fibroblast growth factors (FGF) and fibroblast growth factor receptor 2 (FGFR2)-activating mutations blocks mineralization and induces apoptosis in osteoblasts. J Cell Biol 149: 1297-1308.

Marie, P.J. (2012) Fibroblast growth factor signaling controlling bone formation: an update. Gene 498: 1-4.

-Marie, P.J., H. Miraoui, N. Severe (2012) FGF/ FGFR signaling in bone formation: progress and perspectives. Growth Factors 30: 117123.

Miraoui, H., P.J. Marie (2010) Fibroblast growth factor receptor signaling crosstalk in skeletogenesis. Sci Signal 3: re9.
Morito, A., Y. Kida, K. Suzuki, K. Inoue, N. Kuroda, K. Gomi, T. Arai, T. Sato (2009) Effects of basic fibroblast growth factor on the development of the stem cell properties of human dental pulp cells. Arch Histol Cytol 72: 51-64.

Mulrooney, J.P., T. Hong, L.B. Grabel (2001) Serine 785 phosphorylation of the betal cytoplasmic domain modulates beta1A-integrindependent functions. J Cell Sci 114: 25252533.

Nakao, K., M. Itoh, Y. Tomita, Y. Tomooka, T. Tsuji (2004) FGF-2 potently induces both proliferation and DSP expression in collagen type I gel cultures of adult incisor immature pulp cells. Biochem Biophys Res Commun 325: 1052-1059.

Nakayama, Y., L. Yang, H. Takai, H. Kaneko, Y. Abiko, Y. Ogata (2012) Fibroblast growth factor 2 and forskolin induce mineralization-associated genes in two kinds of osteoblast-like cells. J Oral Sci 54: 251-259.

Osathanon, T., N. Nowwarote, P. Pavasant (2011) Basic fibroblast growth factor inhibits mineralization but induces neuronal differentiation by human dental pulp stem cells through a FGFR and PLCgamma signaling pathway. J Cell Biochem 112: 1807-1816.

Priam, F., V. Ronco, M. Locker, K. Bourd, M. Bonnefoix, T. Duchene, J. Bitard, T. Wurtz, O. Kellermann, M. Goldberg, A. Poliard (2005) New cellular models for tracking the odontoblast phenotype. Arch Oral Biol 50: 271-277.

Roberts-Clark, D.J., A.J. Smith (2000) Angiogenic growth factors in human dentine matrix. Arch Oral Biol 45: 1013-1016.
Shimabukuro, Y., M. Ueda, M. Ozasa, J. Anzai, M. Takedachi, M. Yanagita, M. Ito, T. Hashikawa, S. Yamada, S. Murakami (2009) Fibroblast growth factor-2 regulates the cell function of human dental pulp cells. J Endod 35: 1529-1535.

Smith, A.J., B.A. Scheven, Y. Takahashi, J.L. Ferracane, R.M. Shelton, P.R. Cooper (2012) Dentine as a bioactive extracellular matrix. Arch Oral Biol 57: 109-121.

Suzuki, T., C.H. Lee, M. Chen, W. Zhao, S.Y. Fu, J.J. Qi, G. Chotkowski, S.B. Eisig, A. Wong, J.J. Mao (2011) Induced migration of dental pulp stem cells for in vivo pulp regeneration. J Dent Res 90: 1013-1018.

Tsuboi, T., S. Mizutani, M. Nakano, K. Hirukawa, A. Togari (2003) FGF-2 regulates enamel and dentine formation in mouse tooth germ. Calcif Tissue Int 73: 496-501.

Wu, J., G.T. Huang, W. He, P. Wang, Z. Tong, Q. Jia, L. Dong, Z. Niu, L. Ni (2012) Basic fibroblast growth factor enhances stemness of human stem cells from the apical papilla. J Endod 38: 614-622.

Xiao, L., A. Esliger, M.M. Hurley (2013) Nuclear fibroblast growth factor 2 (FGF2) isoforms inhibit bone marrow stromal cell mineralization through FGF23/FGFR/MAPK in vitro. J Bone Miner Res 28: 35-45.

Xiao, L., P. Liu, X. Li, T. Doetschman, J.D. Coffin, H. Drissi, M.M. Hurley (2009) Exported 18$\mathrm{kDa}$ isoform of fibroblast growth factor- 2 is a critical determinant of bone mass in mice. J Biol Chem 284: 3170-3182.

Yamachika, E., H. Tsujigiwa, M. Matsubara, Y. Hirata, K. Kita, K. Takabatake, N. Mizukawa, Y. Kaneda, H. Nagatsuka, S. Iida (2012) Basic fibroblast growth factor supports expansion of mouse compact bone-derived mesenchymal stem cells (MSCs) and regeneration of bone from MSC in vivo. J Mol Histol 43: $223-$ 233 